\title{
Hyperholomorphic functions and hyper-conjugate harmonic functions of octonion variables
}

\author{
Su Jin Lim and Kwang Ho Shon*
}

\section{"Correspondence:}

khshon@pusan.ac.kr

Department of Mathematics, Pusan

National University, Busan, 609-735, Korea

\begin{abstract}
We represent hyperholomorphic functions on octonionic function theory and octonionic differential operators. We research the properties of hyperholomorphic functions, hyper-conjugate harmonic functions and the integral calculus of hyperholomorphic functions by octonion forms.
\end{abstract}

MSC: 32A99; 30G35; 32W50; $11 \mathrm{E} 88$

Keywords: hyperholomorphic function; Clifford analysis; quaternion; octonion; pseudoconvex domain

\section{Introduction}

The octonions in Clifford algebra are a normed division algebra with eight dimensions over the real numbers larger than the quaternions. The octonions are non-commutative and non-associative but satisfy a weaker form of associativity. The octonions were discovered in 1843 by John T. Graves and constructed in 1845 by A. Cayley. They are referred to as Cayley numbers or the Cayley algebra. The octonions have been applied in fields such as string theory, special theory of relativity and quantum theory. Dentoni and Sce [1] gave a definition of octonionic regular functions and several properties of octonionic regular functions in 1973.

In 2004 and 2006, Kajiwara, Li and Shon [2, 3] obtained some results for the regeneration in complex, quaternion and Clifford analysis, and for the inhomogeneous CauchyRiemann system of quaternion and Clifford analysis in ellipsoid.

In 2011, Koriyama and Nôno [4] gave three regularities (HK-holomorphy, HFholomorphy, $\mathrm{H}_{2}$-holomorphy) of octonionic functions based on holomorphic mappings in a domain in $\mathbf{C}^{4}$. Naser [5] and Nôno [6,7] gave some properties of quaternionic hyperholomorphic functions. For any complex harmonic function $f_{1}$ in a domain of holomorphy $D$ in $\mathbf{C}^{2}$, we can find a function $f_{2}$ such that $f_{1}+f_{2} j$ will be a function hyperholomorphic in $D$ and the Cauchy theorem of hyperholomorphic functions in quaternion analysis. The aim of this paper is to define hyperholomorphic functions with octonion variables in $\mathbf{C}^{4}$ and investigate the properties of the hyperholomorphic functions of octonion variables. We give the condition of harmonicity in $\mathbf{C}^{4}$. Then for any complex-valued functions $g_{1}(z)$ and $g_{2}(z)$ satisfying the condition of harmonicity in a pseudoconvex domain $\Omega$ in $\mathbf{C}^{4}$, we can find hyper-conjugate harmonic functions $g_{3}(z)$ and $g_{4}(z)$, respectively, on $\Omega$ such that 
$g(z)=g_{1}(z)+g_{2}(z) e_{2}+g_{3}(z) e_{4}+g_{4}(z) e_{6}$ is a hyperholomorphic function on $\Omega$. Also, we investigate the Cauchy theorem of hyperholomorphic functions in octonion analysis.

\section{Preliminaries}

The field $\mathcal{O} \cong \mathbf{C}^{4}$ of octonions

$$
z=x_{0}+\sum_{i=0}^{7} e_{i} x_{i}, \quad x_{i}(i=0, \ldots, 7) \in \mathbf{R}
$$

is an eight-dimensional non-commutative and non-associative $\mathbf{R}$-field generated by eight base elements $e_{0}, e_{1}, e_{2}, e_{3}, e_{4}, e_{5}, e_{6}$ and $e_{7}$ with the following non-commutative multiplication rules:

$$
\begin{array}{ll}
e_{i}^{2}=-1, & e_{i} e_{j}=-e_{j} e_{i}, \quad e_{i} e_{j} e_{k}=e_{i}\left(e_{j} e_{k}\right) \quad(i \neq j \neq k, i \neq 0, j \neq 0, k \neq 0), \\
e_{1} e_{2}=e_{3}, & e_{3} e_{5}=e_{6}, \quad e_{6} e_{7}=e_{1}, \quad e_{1} e_{4}=e_{5}, \\
e_{5} e_{7}=e_{2}, & e_{2} e_{6}=e_{4}, \quad e_{4} e_{7}=e_{3} .
\end{array}
$$

The element $e_{0}$ is the identities of $\mathcal{O}$ and $e_{1}$ identifies the imaginary unit $\sqrt{-1}$ in the $\mathbf{C}$ field of complex numbers. An octonion $z$ given by (1) is regarded as $z=z_{1}+z_{2} e_{2}+z_{3} e_{4}+$ $z_{4} e_{6} \in \mathcal{O}$, where $z_{1}:=x_{0}+e_{1} x_{1}, z_{2}:=x_{2}+e_{1} x_{3}, z_{3}:=x_{4}+e_{1} x_{5}$ and $z_{4}:=x_{6}+e_{1} x_{7}$ are complex numbers in $\mathbf{C}$. Thus, we identify $\mathcal{O}$ with $\mathbf{C}^{4}$.

For the equation $z^{3}+8=0$ in the complex plane $\mathbf{C}$, the three solutions are $-2,1+\sqrt{3} i$, $1-\sqrt{3} i$ in $\mathbf{C}$.

In the octonion $\mathcal{O}$, the equation has solutions whose forms are as follows:

$$
z=a+b e_{1}+c e_{2}+d e_{3}+e e_{4}+f e_{5}+g e_{6}+h e_{7} \quad(a, b, c, d, e, f, g, h \in \mathbf{R}) .
$$

Then the equation satisfies $z^{3}=\left(a^{3}-3 a b^{2}-3 a c^{2}-3 a d^{2}-3 a e^{2}-3 a f^{2}-3 a g^{2}-3 a h^{2}\right)+$ $\left(3 a^{2} b-b^{3}-b c^{2}-b d^{2}-b e^{2}-b f^{2}-b g^{2}-b h^{2}\right) e_{1}+\left(3 a^{2} c-b^{2} c-c^{3}-c d^{2}-c e^{2}-c f^{2}-\right.$ $\left.c g^{2}-c h^{2}\right) e_{2}+\left(3 a^{2} d-b^{2} d-c^{2} d-d^{3}-d e^{2}-d f^{2}-d g^{2}-d h^{2}\right) e_{3}+\left(3 a^{2} e-b^{2} e-c^{2} e-d^{2} e-\right.$ $\left.e^{3}-e f^{2}-e g^{2}-e h^{2}\right) e_{4}+\left(3 a^{2} f-b^{2} f-c^{2} f-d^{2} f-e^{2} f-f^{3}-f g^{2}-f h^{2}\right) e_{5}+\left(3 a^{2} g-b^{2} g-\right.$ $\left.c^{2} g-d^{2} g-e^{2} g-f^{2} g-g^{3}-g h^{2}\right) e_{6}+\left(3 a^{2} h-b^{2} h-c^{2} h-d^{2} h-e^{2} h-f^{2} h-g^{2} h-h^{3}\right) e_{7}$. That is, $a^{3}-3 a b^{2}-3 a c^{2}-3 a d^{2}-3 a e^{2}-3 a f^{2}-3 a g^{2}-3 a h^{2}=-8,3 a^{2} b-b^{3}-b c^{2}-b d^{2}-$ $b e^{2}-b f^{2}-b g^{2}-b h^{2}=0,3 a^{2} c-b^{2} c-c^{3}-c d^{2}-c e^{2}-c f^{2}-c g^{2}-c h^{2}=0,3 a^{2} d-b^{2} d-$ $c^{2} d-d^{3}-d e^{2}-d f^{2}-d g^{2}-d h^{2}=0,3 a^{2} e-b^{2} e-c^{2} e-d^{2} e-e^{3}-e f^{2}-e g^{2}-e h^{2}=0$, $3 a^{2} f-b^{2} f-c^{2} f-d^{2} f-e^{2} f-f^{3}-f g^{2}-f h^{2}=0,3 a^{2} g-b^{2} g-c^{2} g-d^{2} g-e^{2} g-f^{2} g-g^{3}-$ $g h^{2}=0,3 a^{2} h-b^{2} h-c^{2} h-d^{2} h-e^{2} h-f^{2} h-g^{2} h-h^{3}=0$. This means that the equation has infinitely many solutions

$$
z=1+b e_{1}+c e_{2}+d e_{3}+e e_{4}+f e_{5}+g e_{6}+h e_{7}
$$

with $b^{2}+c^{2}+d^{2}+e^{2}+f^{2}+g^{2}+h^{2}=3$ in $\mathcal{O}$.

For two octonions $z=\sum_{i=0}^{7} e_{i} x_{i}$ and $w=\sum_{i=0}^{7} e_{i} y_{i}$, the inner product $(z, w)$ is defined as follows:

$$
(z, w):=\sum_{i=0}^{7} x_{i} y_{i}
$$


Also, the octonionic conjugation $z^{*}$, the absolute value $|z|$ of $z$ and an inverse $z^{-1}$ of $\mathrm{z}$ in $\mathcal{O}$ are defined, respectively, by

$$
z^{*}=x_{0}-\sum_{i=1}^{7} e_{i} x_{i}, \quad|z|=\sqrt{\left|z_{1}\right|^{2}+\left|z_{2}\right|^{2}+\left|z_{3}\right|^{2}+\left|z_{4}\right|^{2}}, \quad z^{-1}=\frac{z^{*}}{|z|^{2}} \quad(z \neq 0) .
$$

Thus, the octonion $z \in \mathcal{O}$ and the octonion conjugation $z^{*} \in \mathcal{O}$ have the following forms:

$$
z=x_{0}+e_{1} x_{1}+e_{2} x_{2}+e_{3} x_{3}+e_{4} x_{4}+e_{5} x_{5}+e_{6} x_{6}+e_{7} x_{7}=z_{1}+z_{2} e_{2}+z_{3} e_{4}+z_{4} e_{6}
$$

and

$$
z^{*}=x_{0}-e_{1} x_{1}-e_{2} x_{2}-e_{3} x_{3}-e_{4} x_{4}-e_{5} x_{5}-e_{6} x_{6}-e_{7} x_{7}=\overline{z_{1}}-z_{2} e_{2}-z_{3} e_{4}-z_{4} e_{6},
$$

where $z_{1}=x_{0}+e_{1} x_{1}, z_{2}=x_{2}+e_{1} x_{3}, z_{3}=x_{4}+e_{1} x_{5}$ and $z_{4}=x_{6}+e_{1} x_{7}$.

We use the following differential operators:

$$
\begin{array}{ll}
D_{\alpha}=\frac{\partial}{\partial z_{1}}-e_{2} \frac{\partial}{\partial \overline{z_{2}}}, & D_{\alpha}^{*}=\frac{\partial}{\partial \overline{z_{1}}}+e_{2} \frac{\partial}{\partial \overline{z_{2}}}, \\
D_{\beta}=\frac{\partial}{\partial z_{3}}-e_{2} \frac{\partial}{\partial \overline{z_{4}}}, & D_{\beta}^{*}=\frac{\partial}{\partial \overline{z_{3}}}+e_{2} \frac{\partial}{\partial \overline{z_{4}}},
\end{array}
$$

where $\partial / \partial z_{j}, \partial / \partial \overline{z_{j}}(j=1,2,3,4)$ are usual differential operators used in complex analysis. And we use the following octonionic differential operators:

$$
D:=D_{\alpha}-e_{4} D_{\beta}^{*}, \quad D^{\star}:=D_{\alpha}^{*}+e_{4} D_{\beta}^{*} \text {. }
$$

The operator

$$
\begin{aligned}
D D^{\star} & =\frac{\partial^{2}}{\partial z_{1} \partial \overline{z_{1}}}+\frac{\partial^{2}}{\partial z_{2} \partial \overline{z_{2}}}+\frac{\partial^{2}}{\partial z_{3} \partial \overline{z_{3}}}+\frac{\partial^{2}}{\partial z_{4} \partial \overline{z_{4}}} \\
& =\frac{1}{4}\left(\frac{\partial^{2}}{\partial x_{0}^{2}}+\frac{\partial^{2}}{\partial x_{1}^{2}}+\frac{\partial^{2}}{\partial x_{2}^{2}}+\frac{\partial^{2}}{\partial x_{3}^{2}}+\frac{\partial^{2}}{\partial x_{4}^{2}}+\frac{\partial^{2}}{\partial x_{5}^{2}}+\frac{\partial^{2}}{\partial x_{6}^{2}}+\frac{\partial^{2}}{\partial x_{7}^{2}}\right)
\end{aligned}
$$

is the usual complex Laplacian $\Delta$.

\section{Some properties of hyperholomorphic functions on $\mathcal{O}$}

Let $\Omega$ be an open set in $\mathbf{C}^{4}$. The function $g(z)$ is defined by the following form in $\Omega$ with value in $\mathcal{O}$ :

$$
g(z)=g_{1}(z)+g_{2}(z) e_{2}+g_{3}(z) e_{4}+g_{4}(z) e_{6}=\left\{g_{1}(z)+g_{3}(z) e_{4}\right\}+\left\{g_{2}(z)+g_{4}(z) e_{4}\right\} e_{2},
$$

where $z=\left(z_{1}, z_{2}, z_{3}, z_{4}\right)$ and $g_{1}(z), g_{2}(z), g_{3}(z)$ and $g_{4}(z)$ are complex-valued functions.

Definition 3.1 Let $\Omega$ be an open set in $\mathrm{C}^{4}$. A function $g(z)$ is said to be $\mathrm{L}(\mathrm{R})$ hyperholomorphic in $\Omega$ if the following two conditions are satisfied:

(a) $g_{k}(z)(k=1,2,3,4)$ are continuously differential functions in $\Omega$, and 
(b)

$$
D^{\star} g=0 \quad\left(g D^{\star}=0\right) \quad \text { in } \Omega .
$$

When we deal with an L-hyperholomorphic function $g(z)$ in $\Omega \subset \mathbf{C}^{4}$, for simplicity, we often say that $g(z)$ is a hyperholomorphic function in $\Omega \subset \mathbf{C}^{4}$.

Equation (2) is applied to $g(z)$ as follows:

$$
\begin{aligned}
D^{\star} g= & \left(D_{\alpha}^{*}+e_{4} D_{\beta}^{*}\right)\left(g_{1}(z)+g_{2}(z) e_{2}+g_{3}(z) e_{4}+g_{4}(z) e_{6}\right) \\
= & \left(D_{\alpha}^{*} g_{1}-D_{\beta} \overline{g_{3}}\right)+\left(D_{\alpha}^{*} g_{2}-D_{\beta} \overline{g_{4}}\right) e_{2} \\
& +\left(D_{\alpha}^{*} g_{3}+D_{\beta} \overline{g_{1}}\right) e_{4}+\left(D_{\alpha}^{*} g_{4}+D_{\beta} \overline{g_{2}}\right) e_{6} .
\end{aligned}
$$

If the following equations:

$$
D_{\alpha}^{*} g_{1}=D_{\beta} \overline{g_{3}}, \quad D_{\alpha}^{*} g_{2}=D_{\beta} \overline{g_{4}}, \quad D_{\alpha}^{*} g_{3}=-D_{\beta} \overline{g_{1}}, \quad D_{\alpha}^{*} g_{4}=-D_{\beta} \overline{g_{2}}
$$

are satisfied, the function $g(z)$ is a hyperholomorphic function in $\Omega$. The equations in (3) are the corresponding o-Cauchy-Riemann equations in $\mathbf{C}^{4}$.

Remark 3.2 We redefine equations (3) as follows:

$$
\begin{array}{llll}
\frac{\partial g_{1}}{\partial \overline{z_{1}}}=\frac{\partial \overline{g_{3}}}{\partial z_{3}}, & \frac{\partial g_{1}}{\partial \overline{z_{2}}}=-\frac{\partial \overline{g_{3}}}{\partial \overline{z_{4}}}, & \frac{\partial g_{3}}{\partial \overline{z_{1}}}=-\frac{\partial \overline{g_{1}}}{\partial z_{3}}, & \frac{\partial g_{3}}{\partial \overline{z_{2}}}=\frac{\partial \overline{g_{1}}}{\partial \overline{z_{4}}}, \\
\frac{\partial g_{2}}{\partial \overline{z_{1}}}=\frac{\partial \overline{g_{4}}}{\partial z_{3}}, & \frac{\partial g_{2}}{\partial \overline{z_{2}}}=-\frac{\partial \overline{g_{4}}}{\partial \overline{z_{4}}}, & \frac{\partial g_{4}}{\partial \overline{z_{1}}}=-\frac{\partial \overline{g_{2}}}{\partial z_{3}}, & \frac{\partial g_{4}}{\partial \overline{z_{2}}}=\frac{\partial \overline{g_{2}}}{\partial \overline{z_{4}}} .
\end{array}
$$

We call that equations (4) are the condition of harmonicity.

Remark 3.3 We redefine equations (4) in $\mathbf{R}^{8}$ as follows:

$$
\begin{array}{ll}
\frac{\partial u_{0}}{\partial x_{0}}-\frac{\partial u_{1}}{\partial x_{1}}-\frac{\partial u_{4}}{\partial x_{4}}+\frac{\partial u_{5}}{\partial x_{5}}=0, & \frac{\partial u_{1}}{\partial x_{0}}+\frac{\partial u_{0}}{\partial x_{1}}+\frac{\partial u_{5}}{\partial x_{4}}+\frac{\partial u_{4}}{\partial x_{5}}=0, \\
\frac{\partial u_{0}}{\partial x_{2}}-\frac{\partial u_{1}}{\partial x_{3}}+\frac{\partial u_{4}}{\partial x_{6}}+\frac{\partial u_{5}}{\partial x_{7}}=0, & \frac{\partial u_{1}}{\partial x_{2}}+\frac{\partial u_{0}}{\partial x_{3}}-\frac{\partial u_{5}}{\partial x_{6}}+\frac{\partial u_{4}}{\partial x_{7}}=0, \\
\frac{\partial u_{2}}{\partial x_{0}}-\frac{\partial u_{3}}{\partial x_{1}}-\frac{\partial u_{6}}{\partial x_{4}}+\frac{\partial u_{7}}{\partial x_{5}}=0, & \frac{\partial u_{3}}{\partial x_{0}}+\frac{\partial u_{2}}{\partial x_{1}}+\frac{\partial u_{7}}{\partial x_{4}}+\frac{\partial u_{6}}{\partial x_{5}}=0, \\
\frac{\partial u_{2}}{\partial x_{2}}-\frac{\partial u_{3}}{\partial x_{3}}+\frac{\partial u_{6}}{\partial x_{6}}+\frac{\partial u_{7}}{\partial x_{7}}=0, & \frac{\partial u_{3}}{\partial x_{2}}+\frac{\partial u_{2}}{\partial x_{3}}-\frac{\partial u_{7}}{\partial x_{6}}+\frac{\partial u_{6}}{\partial x_{7}}=0, \\
\frac{\partial u_{4}}{\partial x_{0}}-\frac{\partial u_{5}}{\partial x_{1}}+\frac{\partial u_{0}}{\partial x_{4}}-\frac{\partial u_{1}}{\partial x_{5}}=0, & \frac{\partial u_{5}}{\partial x_{0}}+\frac{\partial u_{4}}{\partial x_{1}}-\frac{\partial u_{1}}{\partial x_{4}}-\frac{\partial u_{0}}{\partial x_{5}}=0, \\
\frac{\partial u_{4}}{\partial x_{2}}-\frac{\partial u_{5}}{\partial x_{3}}-\frac{\partial u_{0}}{\partial x_{6}}-\frac{\partial u_{1}}{\partial x_{7}}=0, & \frac{\partial u_{5}}{\partial x_{2}}+\frac{\partial u_{4}}{\partial x_{3}}+\frac{\partial u_{1}}{\partial x_{6}}-\frac{\partial u_{0}}{\partial x_{7}}=0, \\
\frac{\partial u_{6}}{\partial x_{0}}-\frac{\partial u_{7}}{\partial x_{1}}+\frac{\partial u_{2}}{\partial x_{4}}-\frac{\partial u_{3}}{\partial x_{5}}=0, & \frac{\partial u_{7}}{\partial x_{0}}+\frac{\partial u_{6}}{\partial x_{1}}-\frac{\partial u_{3}}{\partial x_{4}}-\frac{\partial u_{2}}{\partial x_{5}}=0, \\
\frac{\partial u_{6}}{\partial x_{2}}-\frac{\partial u_{7}}{\partial x_{3}}-\frac{\partial u_{2}}{\partial x_{6}}-\frac{\partial u_{3}}{\partial x_{7}}=0, & \frac{\partial u_{7}}{\partial x_{2}}+\frac{\partial u_{6}}{\partial x_{3}}+\frac{\partial u_{3}}{\partial x_{6}}-\frac{\partial u_{2}}{\partial x_{7}}=0,
\end{array}
$$


where $g_{1}=u_{0}+e_{1} u_{1}, g_{2}=u_{2}+e_{1} u_{3}, g_{3}=u_{4}+e_{1} u_{5}$ and $g_{4}=u_{6}+e_{1} u_{7}$ for real-valued functions $u_{i}(i=0, \ldots, 7)$.

\section{Lemma 3.4}

(i) If the function $g(z)$ is hyperholomorphic in an open set $\Omega$ in $\mathbf{C}^{4}$, then the functions $g_{1}(z), g_{2}(z), g_{3}(z)$ and $g_{4}(z)$ are of class $\mathcal{C}^{\infty}$ in $\Omega$.

(ii) If the function $g(z)$ satisfies the condition of harmonicity in an open set $\Omega$ in $\mathbf{C}^{4}$, then the functions $g_{1}(z), g_{2}(z), g_{3}(z)$ and $g_{4}(z)$ are harmonic in $\Omega$.

Proof We have

$$
\begin{aligned}
D D^{\star} g_{1} & =\frac{\partial^{2} g_{1}}{\partial z_{1} \partial \overline{z_{1}}}+\frac{\partial^{2} g_{1}}{\partial z_{2} \partial \overline{z_{2}}}+\frac{\partial^{2} g_{1}}{\partial z_{3} \partial \overline{z_{3}}}+\frac{\partial^{2} g_{1}}{\partial z_{4} \partial \overline{z_{4}}} \\
& =\frac{\partial}{\partial z_{1}}\left(\frac{\partial \overline{g_{3}}}{\partial z_{3}}\right)+\frac{\partial}{\partial z_{2}}\left(-\frac{\partial \overline{g_{3}}}{\partial \overline{z_{4}}}\right)+\frac{\partial}{\partial z_{3}}\left(-\frac{\partial \overline{g_{3}}}{\partial z_{1}}\right)+\frac{\partial}{\partial \overline{z_{4}}}\left(\frac{\partial \overline{g_{3}}}{\partial z_{2}}\right) \\
& =0,
\end{aligned}
$$

and the functions $g_{2}, g_{3}$ and $g_{4}$ are proved by a similar method as in the proof of the case of $g_{1}$. And, by (i), $g_{j}(z)(j=1,2,3,4)$ are of class $\mathcal{C}^{\infty}$ functions in $\Omega$.

Definition 3.5 Let $\Omega \subset \mathbf{C}^{n}$ be an open set with a $\mathcal{C}^{2}$ boundary. Let $\Omega=\{z ; \rho(z)<0\}$, where $\rho$ is in $\mathcal{C}^{2}$ in a neighborhood of $\bar{\Omega}$ and $\operatorname{grad} \rho \neq 0$ on $b \Omega$. Then $\Omega$ is pseudoconvex if

$$
\sum_{j, k=1}^{n} \frac{\partial^{2} \rho}{\partial z_{j} \partial \overline{z_{k}}}(z) w_{j} \overline{w_{k}} \geq 0,
$$

for all $z \in b \Omega$ and $w \in \mathbf{C}^{n}$ satisfying $\sum_{j=1}^{n} \frac{\partial \rho}{\partial z_{j}}(z) w_{j}=0$.

Consider an automorphism $\gamma$ :

$$
\left(z_{1}, z_{2}, z_{3}, s_{4}\right)=\gamma\left(z_{1}, z_{2}, z_{3}, z_{4}\right):=\left(z_{1}, z_{2}, z_{3}, \overline{z_{4}}\right)
$$

of $\mathbf{C}^{4}$. A domain $\Omega$ in $\mathbf{C}^{4} \cong \mathcal{O}$ is said to be pseudoconvex with respect to the complex variables $z_{1}, z_{2}, z_{3}, \overline{z_{4}}$, if $\gamma(\Omega)$ is a pseudoconvex domain of the space $\mathbf{C}^{4}$ of four complex variables $z_{1}, z_{2}, z_{3}, s_{4}$ in the sense of complex analysis.

Theorem 3.6 Let $\Omega$ be a domain in $\mathbf{C}^{4} \cong \mathcal{O}$, which is a pseudoconvex domain with respect to the complex variables $z_{1}, z_{2}, z_{3}, \overline{z_{4}}$ and let $g_{1}(z)$ and $g_{2}(z)$ be complex-valued functions of class $\mathcal{C}^{2}$ on $\Omega$ satisfying the condition of harmonicity (4). Then there exist hyper-conjugate harmonic functions $g_{3}(z)$ and $g_{4}(z)$, respectively, of class $\mathcal{C}^{2}$ on $\Omega$ such that $g(z)$ is a hyperholomorphic function on $\Omega$.

Proof We consider the 1-forms and the differential operator on $\gamma(\Omega)$ :

$$
\begin{aligned}
& \psi_{1}:=-\frac{\partial \overline{g_{1}}}{\partial z_{3}} d \overline{z_{1}}+\frac{\partial \overline{g_{1}}}{\partial \overline{z_{4}}} d \overline{z_{2}}+\frac{\partial \overline{g_{1}}}{\partial z_{1}} d \overline{z_{3}}-\frac{\partial \overline{g_{1}}}{\partial z_{2}} d z_{4}, \\
& \psi_{2}:=-\frac{\partial \overline{g_{2}}}{\partial z_{3}} d \overline{z_{1}}+\frac{\partial \overline{g_{2}}}{\partial \overline{z_{4}}} d \overline{z_{2}}+\frac{\partial \overline{g_{2}}}{\partial z_{1}} d \overline{z_{3}}-\frac{\partial \overline{g_{2}}}{\partial z_{2}} d z_{4}
\end{aligned}
$$


and

$$
\delta=\frac{\partial}{\partial \overline{z_{1}}} d \overline{z_{1}}+\frac{\partial}{\partial \overline{z_{2}}} d \overline{z_{2}}+\frac{\partial}{\partial \overline{z_{3}}} d \overline{z_{3}}+\frac{\partial}{\partial z_{4}} d z_{4} .
$$

We operate the operator $\delta$ from the left-hand side of the 1 -forms $\psi_{1}$ and $\psi_{2}$ on $\gamma(\Omega)$ :

$$
\begin{aligned}
\delta \psi_{1}= & \left(\frac{\partial^{2} \overline{g_{1}}}{\partial \overline{z_{1}} \partial \overline{z_{4}}}+\frac{\partial^{2} \overline{g_{1}}}{\partial \overline{z_{2}} \partial z_{3}}\right) d \overline{z_{1}} \wedge d \overline{z_{2}}+\left(\frac{\partial^{2} \overline{g_{1}}}{\partial \overline{z_{1}} \partial z_{1}}+\frac{\partial^{2} \overline{g_{1}}}{\partial \overline{z_{3}} \partial z_{3}}\right) d \overline{z_{1}} \wedge d \overline{z_{3}} \\
& +\left(-\frac{\partial^{2} \overline{g_{1}}}{\partial \overline{z_{1}} \partial z_{2}}+\frac{\partial^{2} \overline{g_{1}}}{\partial z_{4} \partial z_{3}}\right) d \overline{z_{1}} \wedge d z_{4}+\left(\frac{\partial^{2} \overline{g_{1}}}{\partial \overline{z_{2}} \partial z_{1}}-\frac{\partial^{2} \overline{g_{1}}}{\partial \overline{z_{3}} \partial \overline{z_{4}}}\right) d \overline{z_{2}} \wedge d \overline{z_{3}} \\
& +\left(-\frac{\partial^{2} \overline{g_{1}}}{\partial \overline{z_{2}} \partial z_{2}}-\frac{\partial^{2} \overline{g_{1}}}{\partial z_{4} \partial \overline{z_{4}}}\right) d \overline{z_{2}} \wedge d z_{4}+\left(-\frac{\partial^{2} \overline{g_{1}}}{\partial \overline{z_{3}} \partial z_{2}}-\frac{\partial^{2} \overline{g_{1}}}{\partial z_{4} \partial z_{1}}\right) d \overline{z_{3}} \wedge d z_{4}
\end{aligned}
$$

and

$$
\begin{aligned}
\delta \psi_{2}= & \left(\frac{\partial^{2} \overline{g_{2}}}{\partial \overline{z_{1}} \partial \overline{z_{4}}}+\frac{\partial^{2} \overline{g_{2}}}{\partial \overline{z_{2}} \partial z_{3}}\right) d \overline{z_{1}} \wedge d \overline{z_{2}}+\left(\frac{\partial^{2} \overline{g_{2}}}{\partial \overline{z_{1}} \partial z_{1}}+\frac{\partial^{2} \overline{g_{2}}}{\partial \overline{z_{3}} \partial z_{3}}\right) d \overline{z_{1}} \wedge d \overline{z_{3}} \\
& +\left(-\frac{\partial^{2} \overline{g_{2}}}{\partial \overline{z_{1}} \partial z_{2}}+\frac{\partial^{2} \overline{g_{2}}}{\partial z_{4} \partial z_{3}}\right) d \overline{z_{1}} \wedge d z_{4}+\left(\frac{\partial^{2} \overline{g_{2}}}{\partial \overline{z_{2}} \partial z_{1}}-\frac{\partial^{2} \overline{g_{2}}}{\partial \overline{z_{3}} \partial \overline{z_{4}}}\right) d \overline{z_{2}} \wedge d \overline{z_{3}} \\
& +\left(-\frac{\partial^{2} \overline{g_{2}}}{\partial \overline{z_{2}} \partial z_{2}}-\frac{\partial^{2} \overline{g_{2}}}{\partial z_{4} \partial \overline{z_{4}}}\right) d \overline{z_{2}} \wedge d z_{4}+\left(-\frac{\partial^{2} \overline{g_{2}}}{\partial \overline{z_{3}} \partial z_{2}}-\frac{\partial^{2} \overline{g_{2}}}{\partial z_{4} \partial z_{1}}\right) d \overline{z_{3}} \wedge d z_{4} .
\end{aligned}
$$

By the condition of harmonicity (4), all coefficients vanish. From Hörmander [8], the $\delta$ closed forms $\psi_{1}$ and $\psi_{2}$ of $z_{1}, z_{2}, z_{3}, z_{4}$ are $\delta$-exact forms on $\gamma(\Omega)$. Since $\Omega$ is a pseudoconvex domain, there exist hyper-conjugate harmonic functions $g_{3}(z)$ and $g_{4}(z)$ of class $\mathcal{C}^{\infty}$ on $\Omega$ with $\bar{\partial}$-closed forms $\gamma^{-1} \psi_{1}=\bar{\partial} g_{3}(z)$ and $\gamma^{-1} \psi_{2}=\bar{\partial} g_{4}(z)$ on $\Omega$ of $z_{1}, z_{2}, z_{3}, s_{4}$ are $\bar{\partial}$-exact $(0,1)$-forms on $\Omega$ such that $g(z)$ is a hyperholomorphic function on $\Omega$ (see Krantz [9]).

Theorem 3.7 Let $\Omega$ be a domain in $\mathbf{C}^{4} \cong \mathcal{O}$, which is a pseudoconvex domain with respect to the complex variables $z_{1}, z_{2}, z_{3}, \overline{z_{4}}$ and let $J_{1}(z)=g_{1}(z)+g_{3}(z) e_{4}$ be a complexvalued function of class $\mathcal{C}^{2}$ on $\Omega$ satisfying the condition of harmonicity (4). Then there exists a hyper-conjugate harmonic function $J_{2}(z)=g_{2}(z)+g_{4}(z) e_{4}$ of class $\mathcal{C}^{2}$ on $\Omega$ such that $g(z)=J_{1}(z)+J_{2}(z) e_{2}$ is a hyperholomorphic function on $\Omega$.

Proof We consider the 1 -form and the differential operator on $\gamma(\Omega)$ :

$$
\begin{aligned}
\psi:= & \left(\frac{\partial \overline{g_{4}}}{\partial z_{3}}-\frac{\partial \overline{g_{2}}}{\partial z_{3}} e_{4}\right) d \overline{z_{1}}+\left(-\frac{\partial \overline{g_{4}}}{\partial \overline{z_{4}}}+\frac{\partial \overline{g_{2}}}{\partial \overline{z_{4}}} e_{4}\right) d \overline{z_{2}} \\
& +\left(-\frac{\partial \overline{g_{4}}}{\partial z_{1}}+\frac{\partial \overline{g_{2}}}{\partial z_{1}} e_{4}\right) d \overline{z_{3}}+\left(\frac{\partial \overline{g_{4}}}{\partial z_{2}}-\frac{\partial \overline{g_{2}}}{\partial z_{2}} e_{4}\right) d z_{4}
\end{aligned}
$$

and

$$
\delta=\frac{\partial}{\partial \overline{z_{1}}} d \overline{z_{1}}+\frac{\partial}{\partial \overline{z_{2}}} d \overline{z_{2}}+\frac{\partial}{\partial \overline{z_{3}}} d \overline{z_{3}}+\frac{\partial}{\partial z_{4}} d z_{4} .
$$


We operate the operator $\delta$ from the left-hand side of the 1 -form $\psi$ on $\gamma(\Omega)$ :

$$
\begin{aligned}
\delta \psi= & \left\{\left(-\frac{\partial^{2} \overline{g_{4}}}{\partial \overline{z_{1}} \partial \overline{z_{4}}}-\frac{\partial^{2} \overline{g_{4}}}{\partial \overline{z_{2}} \partial z_{3}}\right)+\left(\frac{\partial^{2} \overline{g_{2}}}{\partial \overline{z_{1}} \partial \overline{z_{4}}}+\frac{\partial^{2} \overline{g_{2}}}{\partial \overline{z_{2}} \partial z_{3}}\right) e_{4}\right\} d \overline{z_{1}} \wedge d \overline{z_{2}} \\
& +\left\{\left(-\frac{\partial^{2} \overline{g_{4}}}{\partial \overline{z_{1}} \partial z_{1}}-\frac{\partial^{2} \overline{g_{4}}}{\partial \overline{z_{3}} \partial z_{3}}\right)+\left(\frac{\partial^{2} \overline{g_{2}}}{\partial \overline{z_{1}} \partial z_{1}}+\frac{\partial^{2} \overline{g_{2}}}{\partial \overline{z_{3}} \partial z_{3}}\right) e_{4}\right\} d \overline{z_{1}} \wedge d \overline{z_{3}} \\
& +\left\{\left(\frac{\partial^{2} \overline{g_{4}}}{\partial \overline{z_{1}} \partial z_{2}}-\frac{\partial^{2} \overline{g_{4}}}{\partial z_{4} \partial z_{3}}\right)+\left(-\frac{\partial^{2} \overline{g_{2}}}{\partial \overline{z_{1}} \partial z_{2}}+\frac{\partial^{2} \overline{g_{2}}}{\partial z_{4} \partial z_{3}}\right) e_{4}\right\} d \overline{z_{1}} \wedge d z_{4} \\
& +\left\{\left(-\frac{\partial^{2} \overline{g_{4}}}{\partial \overline{z_{2}} \partial z_{1}}+\frac{\partial^{2} \overline{g_{4}}}{\partial \overline{z_{3}} \partial \overline{z_{4}}}\right)+\left(\frac{\partial^{2} \overline{g_{2}}}{\partial \overline{z_{2}} \partial z_{1}}-\frac{\partial^{2} \overline{g_{2}}}{\partial \overline{z_{3}} \partial \overline{z_{4}}}\right) e_{4}\right\} d \overline{z_{2}} \wedge d \overline{z_{3}} \\
& +\left\{\left(\frac{\partial^{2} \overline{g_{4}}}{\partial \overline{z_{2}} \partial z_{2}}+\frac{\partial^{2} \overline{g_{4}}}{\partial z_{4} \partial \overline{z_{4}}}\right)+\left(-\frac{\partial^{2} \overline{g_{2}}}{\partial \overline{z_{2}} \partial z_{2}}-\frac{\partial^{2} \overline{g_{2}}}{\partial z_{4} \partial \overline{z_{4}}}\right) e_{4}\right\} d \overline{z_{2}} \wedge d z_{4} \\
& +\left\{\left(\frac{\partial^{2} \overline{g_{4}}}{\partial \overline{z_{3}} \partial z_{2}}+\frac{\partial^{2} \overline{g_{4}}}{\partial z_{4} \partial z_{1}}\right)+\left(-\frac{\partial^{2} \overline{g_{2}}}{\partial \overline{z_{3}} \partial z_{2}}-\frac{\partial^{2} \overline{g_{2}}}{\partial z_{4} \partial z_{1}}\right) e_{4}\right\} d \overline{z_{3}} \wedge d z_{4} .
\end{aligned}
$$

By the same method as the proof of Theorem 3.6, our result is proved.

Theorem 3.8 Let $g(z)$ be a hyperholomorphic function in a domain $G$ of $\mathcal{O}$ and let

$$
\begin{aligned}
\tau= & d z_{1} \wedge d z_{2} \wedge d z_{3} \wedge d z_{4} \wedge d \overline{z_{2}} \wedge d \overline{z_{3}} \wedge d \overline{z_{4}} \\
& +d z_{1} \wedge d z_{2} \wedge d z_{3} \wedge d z_{4} \wedge d \overline{z_{1}} \wedge d \overline{z_{3}} \wedge d \overline{z_{4}} \\
& -d z_{1} \wedge d z_{2} \wedge d z_{4} \wedge d \overline{z_{1}} \wedge d \overline{z_{2}} \wedge d \overline{z_{3}} \wedge d \overline{z_{4}} e_{4} \\
& -d z_{1} \wedge d z_{2} \wedge d z_{3} \wedge d z_{4} \wedge d \overline{z_{1}} \wedge d \overline{z_{2}} \wedge d \overline{z_{3}} e_{4}
\end{aligned}
$$

Then for any domain $\Omega \subset G$ with smooth boundary $b \Omega$,

$$
\int_{b \Omega} \tau g=0,
$$

where $\tau g$ is the octonion product of the form $\tau$ on the function $g(z)$.

Proof Let

$$
\begin{gathered}
\tau_{(1)}=d z_{1} \wedge d z_{2} \wedge d z_{3} \wedge d z_{4} \wedge d \overline{z_{2}} \wedge d \overline{z_{3}} \wedge d \overline{z_{4}} \\
\tau_{(2)}=d z_{1} \wedge d z_{2} \wedge d z_{3} \wedge d z_{4} \wedge d \overline{z_{1}} \wedge d \overline{z_{3}} \wedge d \overline{z_{4}} \\
\tau_{(3)}=d z_{1} \wedge d z_{2} \wedge d z_{4} \wedge d \overline{z_{1}} \wedge d \overline{z_{2}} \wedge d \overline{z_{3}} \wedge d \overline{z_{4}} \\
\tau_{(4)}=d z_{1} \wedge d z_{2} \wedge d z_{3} \wedge d z_{4} \wedge d \overline{z_{1}} \wedge d \overline{z_{2}} \wedge d \overline{z_{3}}
\end{gathered}
$$

By the rule of octonion multiplications,

$$
\begin{aligned}
\tau g= & \left(\tau_{(1)}+\tau_{(2)}-\tau_{(3)} e_{4}-\tau_{(4)} e_{4}\right)\left(g_{1}+g_{2} e_{2}+g_{3} e_{4}+g_{4} e_{6}\right) \\
= & g_{1} \tau_{(1)}+g_{1} \tau_{(2)}-\overline{g_{1}} \tau_{(3)} e_{4}-\overline{g_{1}} \tau_{(4)} e_{4} \\
& +g_{2} \tau_{(1)} e_{2}+g_{2} \tau_{(2)} e_{2}-\overline{g_{2}} \tau_{(3)} e_{6}-\overline{g_{2}} \tau_{(4)} e_{6}
\end{aligned}
$$




$$
\begin{aligned}
& +g_{3} \tau_{(1)} e_{4}+g_{3} \tau_{(2)} e_{4}+\overline{g_{3}} \tau_{(3)}+\overline{g_{3}} \tau_{(4)} \\
& +g_{4} \tau_{(1)} e_{6}+g_{4} \tau_{(2)} e_{6}+\overline{g_{4}} \tau_{(3)} e_{2}+\overline{g_{4}} \tau_{(4)} e_{2} .
\end{aligned}
$$

Hence,

$$
\begin{aligned}
d(\tau g)= & \left(-\frac{\partial g_{1}}{\partial \overline{z_{1}}}-\frac{\partial g_{1}}{\partial \overline{z_{2}}}+\frac{\partial \overline{g_{3}}}{\partial z_{3}}-\frac{\partial \overline{g_{3}}}{\partial \overline{z_{4}}}\right) d V+\left(-\frac{\partial g_{2}}{\partial \overline{z_{1}}}-\frac{\partial g_{2}}{\partial \overline{z_{2}}}+\frac{\partial \overline{g_{4}}}{\partial z_{3}}-\frac{\partial \overline{g_{4}}}{\partial \overline{z_{4}}}\right) d V e_{2} \\
& +\left(-\frac{\partial \overline{g_{1}}}{\partial z_{3}}+\frac{\partial \overline{g_{1}}}{\partial \overline{z_{4}}}-\frac{\partial g_{3}}{\partial \overline{z_{1}}}-\frac{\partial g_{3}}{\partial \overline{z_{2}}}\right) d V e_{4}+\left(-\frac{\partial \overline{g_{2}}}{\partial z_{3}}+\frac{\partial \overline{g_{2}}}{\partial \overline{z_{4}}}-\frac{\partial g_{4}}{\partial \overline{z_{1}}}-\frac{\partial g_{4}}{\partial \overline{z_{2}}}\right) d V e_{6},
\end{aligned}
$$

where $d V=d z_{1} \wedge d z_{2} \wedge d z_{3} \wedge d z_{4} \wedge d \overline{z_{1}} \wedge d \overline{z_{2}} \wedge d \overline{z_{3}} \wedge d \overline{z_{4}}$, and by the condition of harmonicity (4), $d(\tau g)=0$. By Stoke's theorem, we have

$$
\int_{b \Omega} \tau g=\int_{\Omega} d(\tau g)=0
$$

\section{Competing interests}

The authors declare that they have no competing interests.

\section{Authors' contributions}

All authors read and approved the final manuscript.

\section{Acknowledgements}

The second author was supported by the Basic Science Research Program through the National Research Foundation of Korea (NRF) funded by the Ministry of Education, Science and Technology (2010-0009646), and by the Research Fund Program of Research Institute for Basic Sciences, Pusan National University, Korea, 2012, Project No. RIBS-PNU-2012-101.

Received: 6 November 2012 Accepted: 26 January 2013 Published: 1 March 2013

\section{References}

1. Dentoni, P, Sce, M: Funzioni regolari nell'algebra di Cayley. Rend. Sem. Mat. Univ. Padora 50, 251-267 (1973)

2. Kajiwara, J, Li, XD, Shon, KH: Regeneration in complex, quaternion and Clifford analysis. In: Proc. the 9th International Conf. on Finite or Infinite Dimen, Hanoi, pp. 287-298. Complex Analysis and Appl., vol. 9. Kluwer Academic, Dordrecht (2004)

3. Kajiwara, J, Li, XD, Shon, KH: Function spaces in complex and Clifford analysis, inhomogeneous Cauchy Riemann system of quaternion and Clifford analysis in ellipsoid. In: Proc. the 14th International Conf. on Finite or Infinite Dimen, Hue, pp. 127-155. Complex Analysis and Appl., vol. 14. Hue Univ., Vietnam (2006)

4. Koriyama, H, Nôno, K: On regularities of octonionic functions and holomorphic mappings. Bull. Fukuoka Univ. Edu. Part III 60, 11-28 (2011)

5. Naser, M: Hyperholomorphic functions. Silberian Math. J. 12, 959-968 (1971)

6. Nôno, K: Hyperholomorphic functions of a quaternion variable. Bull. Fukuoka Univ. Edu. 32, 21-37 (1983)

7. Nôno, K: Characterization of domains of holomorphy by the existence of hyper-conjugate harmonic functions. Rev. Roum. Math. Pures Appl. 31(2), 159-161 (1986)

8. Hörmander, L: An Introduction to Complex Analysis in Several Variables. North-Holland, Amsterdam (1966)

9. Krantz, SG: Function Theory of Several Complex Variables. Am. Math. Soc., Providence (2001)

doi:10.1186/1029-242X-2013-77

Cite this article as: Lim and Shon: Hyperholomorphic functions and hyper-conjugate harmonic functions of octonion variables. Journal of Inequalities and Applications 2013 2013:77. 